\title{
Désactivation des défibrillateurs cardioverteurs implantables
}

\author{
Darryl Wan MD, Santabhanu Chakrabarti MBBS MD
}

Citation : CMAJ 2021 June 7;193:E852. doi : 10.1503/cmaj.210327-f

Voir la version anglaise de l'article ici : www.cmaj.ca/lookup/doi/10.1503/cmaj.210327

1

La désactivation des appareils devrait être abordée quand les objectifs du traitement changent

Environ 7000 défibrillateurs cardioverteurs implantables (DCl) sont posés chaque année au Canada pour prévenir la mort subite ${ }^{1}$. Or, à un moment, il se peut que les préférences des patients changent et que les objectifs de soins obéissent à des impératifs liés à la qualité de vie plutôt qu'à la prévention de la mort subite.

2

\section{Laisser les défibrillateurs cardioverteurs}

implantables actifs peut occasionner des douleurs et une souffrance indues

Les chocs administrés par les défibrillateurs cardioverteurs implantables peuvent occasionner de l'anxiété, de la douleur et une crainte des chocs répétitifs anticipés, car jusqu'à $20 \%$ des patients reçoivent des chocs au cours de leurs dernières semaines de vie ${ }^{2}$. La majorité des patients qualifient ces chocs d'intenses, au point de causer une détresse substantielle chez les patients et leurs proches aidants. En présence de maladie en phase terminale, les arythmies ventriculaires peuvent être la manifestation d'une défaillance pluriorganique et les chocs administrés par les défibrillateurs cardioverteurs implantables peuvent occasionner de la douleur, sans pour autant corriger l'anomalie sous-jacente.

\section{La désactivation d'un défibrillateur}

cardioverteur implantable est indolore, non effractive et réversible

La désactivation est faite en contexte clinique, par un membre qualifié de l'équipe responsable des stimulateurs cardiaques ou de la clinique d'arythmie, après discussion avec l'équipe soignante, et avec le consentement du patient ou de la personne désignée comme mandataire. La désactivation ne modifie pas la fonction du stimulateur cardiaque et n'entraîne pas le décès immédiat; elle n'affecte pas immédiatement les symptômes et l'appareil peut être remis en fonction si les objectifs de soins changent.
Si la désactivation programmée n'est pas disponible, un aimant peut être utilisé pour désactiver les chocs

Un aimant de qualité médicale (disponible dans les services d'urgence et les cliniques de stimulateurs cardiaques) peut être fixé à la peau au-dessus du défibrillateur cardioverteur implantable pour désactiver l'administration des chocs (figure 1). Dès que l'aimant est enlevé, l'appareil reprend sa fonction normale.

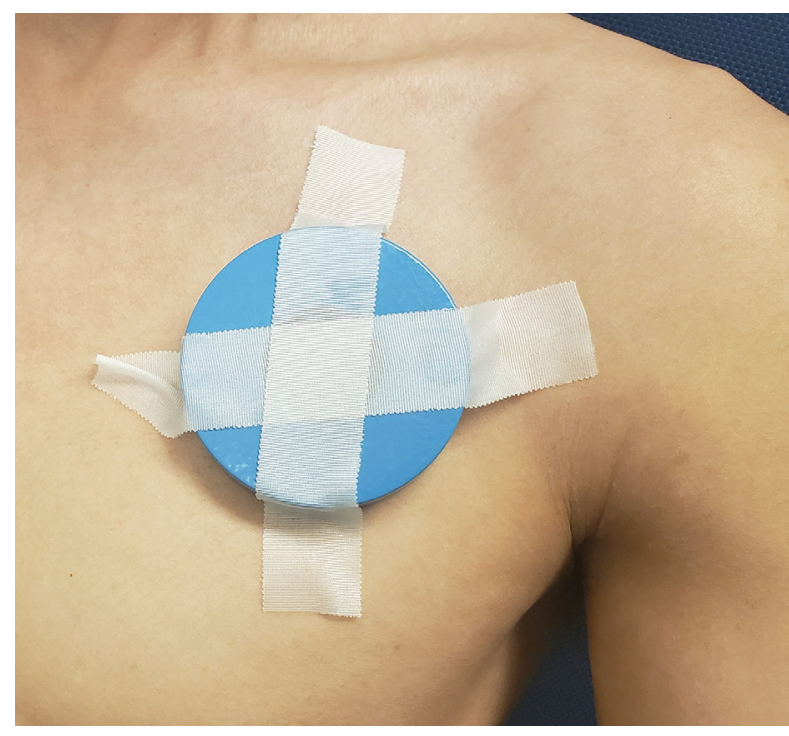

Figure 1 : Aimant fixé en place juste au-dessus de la pochette d'un défibrillateur cardioverteur implantable.

5 La désactivation d'un défibrillateur cardioverteur implantable correspond à un arrêt de traitement Aux plans juridique et éthique, la désactivation de l'appareil ne constitue pas une aide médicale à mourir. II n'y a pas de différence entre le refus d'un défibrillateur cardioverteur implantable et la demande de désactivation de l'appareil par un patient, car tous les patients ont le droit constitutionnel de refuser tout traitement ${ }^{3}$. 


\section{Références}

1. Kelly SE, Campbell D, Duhn LJ, et al. Remote monitoring of cardiovascular implantable electronic devices in Canada: survey of patients and device healthcare professionals. CJC Open 2020 Nov. 19. doi: 10.1016/j.cjco.2020 .11 .010 .

2. Sherazl S, McNitt S, Aktas MK, et al. End-of-life care in patients with implantable cardioverter defibrillators: a MADIT-II substudy. Pacing Clin Electrophysiol 2013;36:1273-9.

3. Lampert R, Hayes DL, Annas GJ, et al. HRS expert consensus statement on the management of cardiovascular implantable electronic devices (CIEDs) in patients nearing end of life or requesting withdrawal of therapy. Heart Rhythm 2010;7:1008-26.

Le JAMC vous invite à soumettre vos textes pour la rubrique « Cinq choses à savoir ... » en ligne à http://mc.manuscriptcentral.com/cmaj.
Intérêts concurrents : Aucun déclaré.

Cet article a été révisé par des pairs.

Affiliations : Département de médecine, Division ce cardiologie, Université de la Colombie-Britannique, Vancouver, C.-B.

Propriété intellectuelle du contenu : Il s'agit d'un article en libre accès distribué conformément aux modalités de la licence Creative Commons Attribution (CC BY-NC-ND 4.0), qui permet l'utilisation, la diffusion et la reproduction de tout médium à la condition que la publication originale soit adéquatement citée, que l'utilisation se fasse à des fins non commerciales (c.-à-d., recherche ou éducation) et qu'aucune modification ni adaptation n'y soit apportée. Voir : https://creativecommons.org/licenses/by-nc-nd/4.0/deed.fr.

Correspondance : Santabhanu Chakrabarti, sChakrabarti@providencehealth.bc.ca 